\title{
Totémisme duel, totémisme pluriel. Un exemple de Nouvelle-Guinée
}

Dual totemism, plural totemism: An example from New Guinea

\section{Bernard Juillerat}

\section{(2) OpenEdition \\ Journals}

Édition électronique

URL : http://journals.openedition.org/span/1570

DOI : 10.4000/span. 1570

ISSN : 2268-1558

Éditeur

École pratique des hautes études. Sciences humaines

\section{Édition imprimée}

Date de publication : 1 décembre 1998

Pagination : 13-106.

ISSN : 0294-7080

\section{Référence électronique}

Bernard Juillerat, «Totémisme duel, totémisme pluriel. Un exemple de Nouvelle-Guinée », Systèmes de pensée en Afrique noire [En ligne], 15 | 1998, mis en ligne le 28 mai 2014, consulté le 19 avril 2019. URL http://journals.openedition.org/span/1570 ; DOI : 10.4000/span.1570 


\section{TOTÉMISME DUEL, TOTÉMISME PLURIEL. \\ Un exemple de Nouvelle-Guinée}

par

\section{Bernard Juillerat}

A l'opposé de la Polynésie ou de l'Australie où la comparaison interne est plus aisée, la Mélanésie rend tout travail comparatif redoutable par sa grande diversité culturelle. Le métissage culturel entre les anciennes cultures non-austronésiennes (remontant à 50000 ans) et les apports mélanésiens plus récents, ainsi que l'isolement relatif des sociétés durant des siècles paraissent responsables de la diversité constatée aujourd'hui (Howells, 1973). Cela explique l'impossibilité où l'on se trouve d'entreprendre de façon efficace une étude comparative des formes de totémisme en Mélanésie. On constate néanmoins que les institutions ou formes de représentations qui s'apparentent, de près ou de loin, à ce qu'il fut convenu de nommer " totémisme " sont principalement attestées en Mélanésie insulaire et, pour la Nouvelle-Guinée, dans les basses terres, c'est-à-dire là où l'influence austronésienne a eu des effets durables (y compris dans des sociétés parlant aujourd'hui des langues non-austronésiennes). L'apparentement symbolique entre groupes sociaux et espèces naturelles y est particulièrement évident dans les organisations à moitiés. Aussi, plutôt que de tenter en vain de mettre en parallèle, par exemple, l'origine des quatre clans trobriandais avec le totémisme des noms de

\section{Totémismes}

Systèmes de pensée en Afrique noire, 15, 1998 
personnes au moyen Sépik (Bateson, 1958, Harrison, 1990), ou encore de comparer entre eux les aspects totémiques des innombrables organisations à moitiés attestées en Mélanésie insulaire et dans les basses terres de Nouvelle-Guinée, j'ai choisi de reprendre mes propres matériaux sur la société yafar du Sépik occidental, même s'ils ont déjà été publiés (Juillerat, 1986, 1992, 1995). La question du totémisme yafar n'avait cependant, jusque là, pas été traitée en tant que telle.

La structure sociale yafar ${ }^{1}$ est caractérisée par une double organisation dualiste (moitiés et semi-moitiés) dans lesquelles se répartissent une quinzaine de patriclans, dont la moitié sont considérés comme anciens. Dans mes travaux précédents, je n'avais qualifié de " totémiques" que les moitiés, alors que pour les patriclans je n'avais parlé que d'emblèmes (figurant des espèces naturelles) peints sur certains masques du rite Yangis; dans la présente discussion, et afin de mettre les deux systèmes en parallèle, je parlerai exceptionnellement de totémisme clanique aussi bien que de totémisme de moitiés. "Totémisme clanique " ne se référera ici qu'aux emblèmes rituels du culte masculin, alors qu'en réalité ce terme conviendrait mieux à la relation que certains clans établissent, dans des mythes tenus secrets (même au sein de la société), entre des espèces naturelles et leurs ancêtres mythiques respectifs. N'ayant pu recueillir qu'un seul de ces mythes bien gardés (celui du clan Sumneri qui situe son origine naturelle dans deux variétés de bambou [Juillerat, $1986: 99]$ ), je ne suis pas à mêrne d'en faire une étude comparative globale. Par contre, les espèces emblématiques ne sont pas considérées comme étant à l'origine du clan, mais seulement liées à lui par des interdits et surtout par un esprit de la croissance (ogomô) individualisé et nommé. Quant à la paire de totems des moitiés, elle renvoie explicitement à la dualité sexuelle et, plus particulièrement, à l'opposition père/mère et au couple divin ancestral. Les semi-moitiés, quant à elles, sont respectivement connotées aîné/cadet pour chaque moitié, mais ne sont pas totémiquement marquées; elles constituent en revanche les unités exogamiques les

${ }^{1}$ Les Yafar forment une petite communauté de 200 membres habitant la région forestière des Border Mountains (Papouasie Nouvelle-Guinée), proche de la frontière avec l'Irian Jaya (Indonésie). Les Yafar vivent dans de petits hameaux et pratiquent l'horticulture itinérante sur brûlis, la cueillette et la chasse (Juillerat, 1986). 
plus larges, les patriclans fonctionnant dans l'alliance comme les groupes échangistes. Si l'on regroupe formellement les deux semi-moitiés " aînées " et les deux semi-moitiés " cadettes " en deux unités opposées, transversales par rapport aux moitiés de genres, on obtient ce que nous nommerons des " fausses moitiés". Enfin, un clan fondateur (appartenant à la semi-moitié " ânée " de chaque moitié) se voit réserver une fonction rituelle particulière incarnant le genre (masculin/féminin) et la partie du cosmos (ciel/terre) associés à sa moitié. Les deux systèmes totémiques (de clans et de moitiés), quoiqu'obéissant à des principes différents, se trouvent indissociablement liés dans la pratique rituelle du culte masculin.

C'est donc au travers de ce culte et de sa réalisation rituelle centrale, Yangis, que l'on pourra appréhender la forme et le contenu du totémisme yafar. Je commencerai par le totémisme de moitiés. Chez les Yafar, la fonction sociale des moitiés reste faible, alors que l'organisation exogamique de la société (fondée, comme on l'a vu, sur les semi-moitiés) a une structure quadripartite. Il y a donc, dans la double organisation dualiste ainsi composée, une imbrication formelle de la fonction sociale et de la fonction symbolique. Le marquage " aîné/cadet " des semi-moitiés reste pour sa part également symbolique, les quatre groupes exogames apparaissant, sur le plan de l'organisation de l'alliance, parfaitement égaux entre eux (leurs seules différences sont celles de leur importance démographique relative), sans distinction entre groupes donneurs ou preneurs. Dans Yangis, ni les semi-moitiés ni les fausses moitiés "aînée/cadette " ne sont retenues. Nous nous retrouvons donc avec une dualité simple, mais dont la richesse symbolique et les connotations renvoyant à l'opposition des sexes ou au couple parental sont redondantes.

Les Yafar font commencer le monde avec un couple ancestral, d'apparence humaine, qui flottait dans l'espace cosmique avant même la formation de la terre et du soleil. Pour respecter leurs noms secrets, je les désigne par leurs initiales : W pour lui, B pour elle. Mais ce couple prend sur terre la forme de deux palmiers, le sagoutier paternel (na) et le cocotier maternel (sa), respectivement totems des moitiés "masculine " et " féminine ". A un second niveau totémique on les retrouve sous la forme de deux clones de sagoutier, la variété afweêêg pour le père originel $W$, la 
variété fenaw pour la mère originelle $\mathrm{B}^{2}$. A leur tour, les versions profanes de certains mythes donnent d'autres espèces végétales dans le but de cacher l'identification aux divinités primordiales, dont l'existence ou les noms ne sont jamais mentionnés ${ }^{3}$. On voit donc que se dégage une hiérarchie des références totémiques, allant du plus profane au plus sacré. Alors que la terre et le ciel se formèrent à partir des sécrétions sexuelles des accouplements primordiaux, B finit par mourir d'une hémorragie ; de ce sang répandu naquirent cependant les deux "fils totémiques ", ifegê (littéralement : " hommes "); ils sont aussi nommés, dans le contexte de Yangis, na yug / sa yug, " nouvelle pousse de sagoutier / nouvelle pousse de cocotier ". Ils figureront, dans Yangis, les deux totems - donc les deux moitiés de la société - renouvelés, mais aussi les totems transformés en êtres humains. Les deux moitiés, masculine/féminine ou paternelle/maternelle, se reproduisent cependant en des fils (et non en un couple frère/sœur), marqués du genre de leur moitié respective. A la mort de la Mère primordiale, son compagnon W se châtra et planta son pénis dans le sol, créant ainsi (selon certaines versions) le premier sagoutier afwêêg. Puis il découpa le corps de $\mathbf{B}$ et en disposa les organes pour créer tout ce qui de l'univers et de la nature n'existait pas encore. Bien que je ne possède pas l'épisode du sagoutier fenaw et seulement un mythe obscur pour le cocotier ${ }^{4}$, on peut penser que ces palmiers sont issus d'un organe du corps maternel. Mais la première partie du corps, découpé et placé dans l'univers, est le sein de B (mamelle unique et centrée), que son compagnon monte suspendre au ciel couchant où il devient le soleil. Puis W aide ses fils, les ifegê, à survivre ; ils sont en effet affaiblis par leur

${ }^{2} \mathrm{~J}$ 'ai montré dans $L$ 'avènement du père qu'on pouvait aussi voir dans les deux variétés de sagoutier deux totems paternels, un pour chaque moitié.

${ }^{3}$ Les noms sont tout au plus murmurés de façon inaudible dans les incantations magiques sur la fertilité.

${ }^{4}$ Ce récit fait naître le cocotier de la métamorphose d'un casoar originel. Cet oiseau apparait en effet comme le premier être de l'univers dans la mythologie waina (voir Gell 1975, Juillerat 1992). 
séjour dans le corps de leur mère ${ }^{5}$ et ils ont faim. Il leur manque en quelque sorte la résistance intrinsèque à leur sexe. C'est pourquoi leur père divin panse leur pénis * fait que de sang " et leur donne un petit arc et une fléchette, signe de leur masculinité et arme de leurs futures chasses. Il s'agit en fait de les détourner du sol où les enfants cherchent instinctivement leur mère, et, en tirant lui-même une flèche épointée, de leur désigner le sein suspendu dans le ciel. Les deux fils tirent à leur tour leurs flèches pour s'approprier le sein qui devient leur premier gibier (premier objet d'investissement). C'est pourquoi, en conclusion du rite Yangis, les deux hommes (peints d'ocre rouge de la tête aux pieds) qui incarnent les ifegê décochent une flèche vers le soleil déclinant.

Yangis est, pour nous et de toute évidence, un rite totémique, c'està-dire un dispositif symbolique assurant le renouvellement d'espèces naturelles, considérées par ailleurs comme l'origine des groupes totémiques (moitiés) ${ }^{6}$. Renouvellement des espèces signifie donc simultanément reproduction des moitiés, donc de la société comme tout. Nous verrons en conclusion comment Yangis met en œuvre, par sa face cachée, un scénario sociogénétique fondé sur une ontogenèse du héros mythorituel. Son but ouvertement déclaré par les Yafar n'est pourtant que d'assurer la croissance des sagoutiers qui foumissent la base de l'alimentation. A ce premier niveau du totémisme de moitiés revendiqué par l'interprétation populaire locale, on aurait donc affaire à un totémisme " utilitaire ", à ce totémisme fonctionnaliste que Lévi-Strauss prend soin d'écarter à propos des thèses de Malinowski. Mais dès que l'on remplace l'opposition duelle afwêê/fenaw par la paire sagoutier/cocotier, directe incarnation du couple parental ancestral, on ne peut plus faire valoir l'argument de Malinowski selon lequel les totems représentent les espèces alimentaires principales dont la société a besoin. En effet, si le sagoutier fournit par sa

5 On peut penser à ce sujet que l'accouchement-hémorragie de $B$ est un acte parthénogénétique plutôt que le résultat de la sexualité, ou qu'il y a pour le moins l'effet d'une domination féminine dans la procréation réduisant le rôle paternel à peu de chose, insignifiance que le dieu compense immédiatement en " initiant "ses fils, en les séparant de la mère parturiente.

"La moitié masculine se désigne par l'expression " enfants du sang du sagoutier"; la moitié féminine se dit " enfants du sang (des fleurs) du cocotier ". D'une façon plus générale, les Yafar se nomment métaphoriquement " enfants du sang ". 
fécule l'aliment quotidien, le cocotier est totalement secondaire sur le plan alimentaire. Ses noix sont rarement cueillies, bues ou râpées pour la consommation. L'opposition entre les deux genres de palmier repose sur d'autres critères. D'abord un critère environnemental, que soulignent les Yafar eux-mêmes : le sagoutier croît en forêt (c'est une espèce spontanée de la Nouvelle-Guinée) alors que le cocotier est exclusivement planté autour des hameaux. Les Yafar disent souvent : " Notre mère reste au village devant la maison, tandis que notre père vit en forêt ". Ensuite un critère morphologique. Le sagoutier ne fleurit qu'une seule fois, avant de mourir, son inflorescence ressemblant à un grand candélabre dressé au sommet de la couronne (contrastant avec les inflorescences latérales et tombantes du cocotier), mais ses fruits sont presque toujours stériles et ce palmier se perpétue grâce à ses rejets spontanés. Au contraire, le cocotier se reproduit sexuellement à partir de ses noix que les Yafar laissent germer dans leurs maisons avant de les repiquer. Les noix renvoient aux seins de la mère lorsqu'elles pendent pleines d'eau, au ventre gestant lorsque, remplies de copra, elles germent au sol. Les fleurs du cocotier symbolisent le lait maternel primordial et constituent en ce sens un interdit alimentaire (bien que non comestibles). Par ailleurs, le tissu infrapétiolaire emballant chaque nouvelle palme est désigné comme un utérus donnant naissance à un fils. Enfin, dernière différence, le sagoutier nourrit les hommes à partir de sa moelle que l'on filtre pour en obtenir le sagou ; c'est pourquoi les Yafar comparent le sagoutier à un sexe viril dressé et rempli de sperme ${ }^{7}$. Le stipe du cocotier ne contient en revanche aucune fécule. On comprend donc qu'il ne s'agit guère de nourriture ici (malgré l'importance alimentaire du sagou), mais que les deux totems symbolisent les appareils reproducteurs et nourriciers de l'homme et de la femme : organe du père, organes de la mère. Le phallus est représenté dans son autorégénération, le corps de la mère dans sa double fonction gestative et nourricière.

La danse nocturne ouvrant le rite Yangis met en scène deux danseurs peints de noir et portant chacun un masque surmonté de palmes de

${ }^{7}$ Sur l'identification du sagou au sperme, voir aussi Gell 1975 
sagoutier ${ }^{8}$ (les feuilles de cocotiers sont l'objet d'un tabou). Leur danse ininterrompue jusqu'à l'aube reprend la copulation fondatrice de W et B dans la nuit cosmique. A l'aube, ces deux personnages sont remplacés par deux autres, nommés " Gelée de sagou ". Alors que ces deux nouveaux protagonistes dansent avec les mêmes masques à feuilles de sagoutier, les deux maîtres rituels des moitiés (Maître du Ciel et Maître de la Terre) fabriquent sur la place une gelée de sagou à partir de fécule provenant des deux clones totémiques. Lorsqu'elle se fige sous l'effet de l'eau bouillante, la gelée devient le double embryon des ifegê dans le ventre maternel. Elle est ensuite projetée en l'air pour figurer l'amorce de la croissance. Tout le reste de la journée sera occupé par la danse d'autres personnages rituels aux corps d'abord noirs, puis couverts de motifs polychromes ; les premiers incarnent les esprits de la croissance des sagoutiers, ogomô, les seconds les fœtus totémiques en gestation associés symboliquement aux poissons de la rivière et désignés par le terme "Poissons ". Contrairement aux masques de la nuit et de l'aube, personnifiant les totems de moitiés, les ogomô et les Poissons portent des masques claniques dotés chacun de son emblème " totémique " propre (voir ci-dessous). Seuls les membres du clan ou leurs enfants de sœurs portent ces masques, au nombre de sept. Les clans entrent donc " dans la danse " sous l'identité de leur esprit ogomô et de leur emblème respectifs, afin de promouvoir la croissance des deux fœtus totémiques. Le double tissu infrapétiolaire de cocotier tendu sur l'armature du masque et ayant reçu, à l'abri du regard des femmes et des enfants, les peintures claniques figure l'utérus de la mère primordiale $B$. Les danseurs ont ainsi la tête enchâssée dans le ventre maternel originel marqué en l'occurrence du signe reconnu de chaque clan. Au terme du rite, en fin d'après-midi", apparaissent les deux ifegê, les fils totémiques libérés du ventre parturient et en quête du sein ; cette quête est accomplie grâce à l'aide de leur père qui, dans le rite, est qualifié de " oncle maternel ". Les deux ifegê portent également un masque à toile peinte, mais il s'agit de motifs abstraits non

${ }^{8}$ Du clone afwêêg pour le danseur de la moitié " masculine ", du clone fenaw pour le danseur de la moitié "féminine".

9 Je résume le rite à l'extrême et saute volontairement les rôles secondaires (voir Juillerat, 1992 ou 1995 et Gell, 1975). 
rapportés à un clan particulier et ne figurant aucune espèce naturelle. Après le défilé des sept masques claniques tout au long du jour, on revient ainsi à la dualité des moitiés. Ce sont alors les corps rouges (ensanglantés) des ifegê qui personnifient les na yug / sa yug, les deux totems renouvelés, donnés en même temps comme " premiers hommes " et socialisés en tant que tels par leur père/oncle maternel avec la contribution de la mère réduite à sa fonction nourricière (sein-soleil). Les « fils totémiques *s'inscrivent dans la dualité des moitiés, mais ne portent plus les masques-sagoutiers de leurs parents : leurs "emblèmes * sont apparentés formellement à ceux des clans, mais ne se rapportent à aucun clan particulier. Ils incarnent l'ensemble de la société.

Avant d'analyser plus en détails cette imbrication des deux totémismes (de moitiés et de clans, duel et pluriel), il faut préciser dans quelle mesure les espèces naturelles totémiques pour les clans paraissent arbitraires, contrairement à celles des moitiés qui renvoient à la dualité sexuelle et parentale. Les sept emblèmes claniques, représentant les espèces interdites (elles ne sont cependant pas toutes des aliments) aux membres du clan, sont les suivantes :

MOITIE

MOITIE MASCULINE

MOITIÉ FÉMININE
CLAN

Clan Wamawneri

Clan Sumneri

Clan Wiyneri

Clan Ifêêg

Clan Menaneri

Clan Biyuneri

Clan Bwasneri

Clan Amisneri

\section{TOTEM}

Jeune casoar

(kwoyrêmp)

Larve bwerinaag

Pigeon yibus

Chouette wêy

? (clan éteint)

Arbre sêbêf

Insecte wank

Serpent ha 


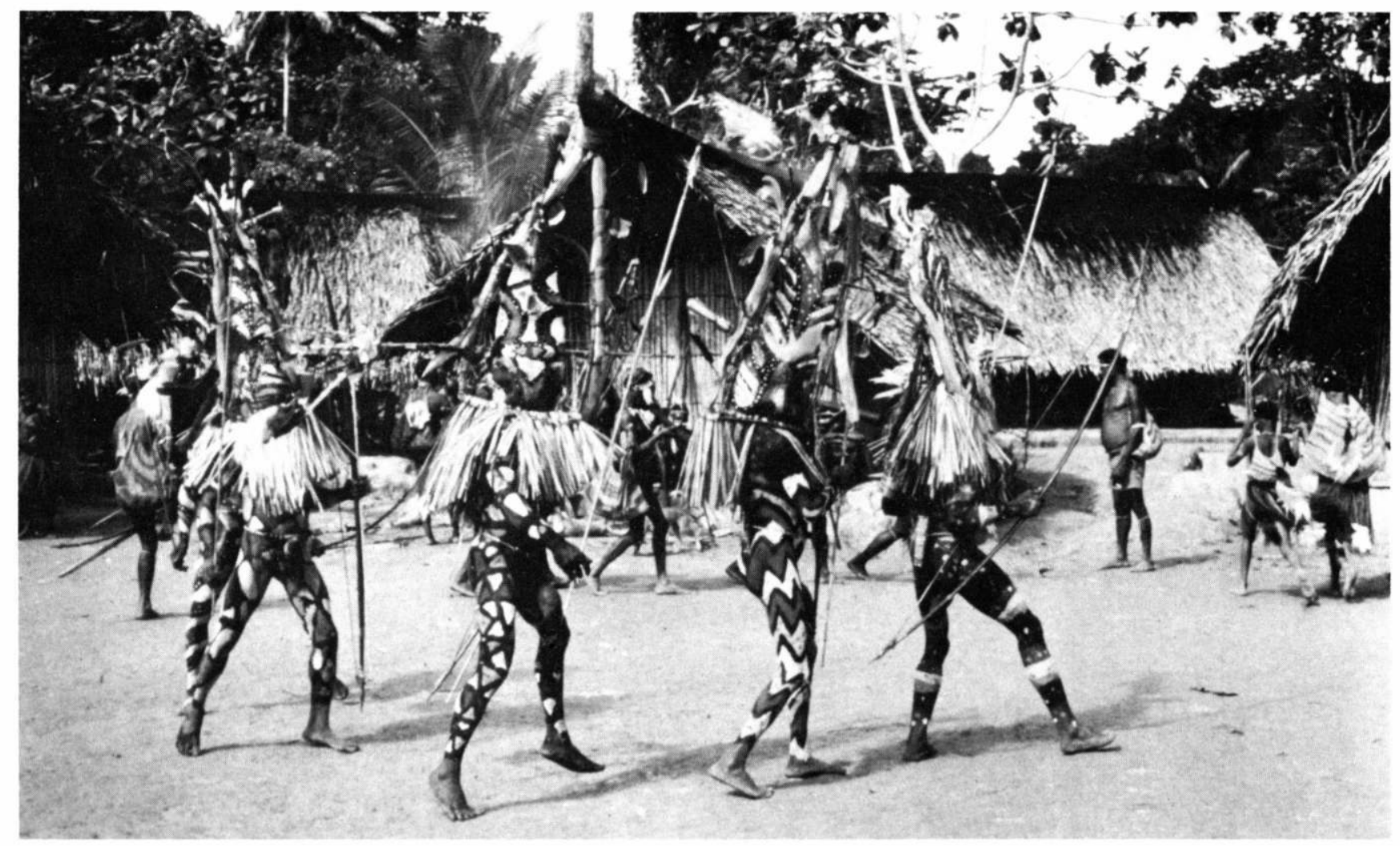

Personnages " Poissons " au corps polychrome portant les masques emblématiques des patriclans. Rites Yangis, Yafar, 1976 (Cliché B. Juillerat) 
Si l'on met à part le clan fondateur Wamawneri et son emblème du jeune casoar (plus précisément les rayures du plumage du jeune casoar : kwoyrêmp), les espèces totémiques ne présentent entre elles aucune cohérence $^{10}$. Dans la séquence de la danse des esprits ogomô, le masque kwoyrêmp est porté par un danseur au corps peint d'ocre rouge marchant devant les autres (peints en noir). Ce privilège réfère généralement, dans les différents cortèges de masques dont le complexe rituel yafar est doté, au statut du jeune chasseur conduisant la communauté tout entière et incarnant son renouvellement générationnel. Ce rôle rituel n'est assumé qu'une seule fois dans la vie d'un homme. Le clan fondateur " masculin " a ici la préséance sur le clan fondateur "féminin ". Dans le contexte de Yangis, on peut supposer que ce rôle personnifie les ifegê dont la sortie sur la place (la naissance) est reportée à la fin de la représentation. Dans la mythologie, le casoar est une figure maternelle (comme dans presque toutes les cultures de Nouvelle-Guinée) et il est en outre directement associé au cocotier, totem-mère (voir supra note 4). Mais il n'a pas de statut totémique en soi. Le masque Wamawneri montre le plumage caractéristique du jeune casoar, dont on peut penser qu'il est ici le fils à naître de la mère-casoar. Les espèces totémiques des six autres clans ne renvoient à ma connaissance à aucun mythe et ne paraissent pas non plus faire système entre eux. L'arbre sêhêf et l'insecte wank ne sont pas des espèces comestibles; le pigeon yibus est de petite taille et n'est pas chassé (il symbolise cependant parfois l'homme, par opposition à la femme représentée par l'oiseau de Paradis). Parmi les groupes de langue waina, voisins des Yafar au nord, Alfred Gell ne fournit aucune liste pour les emblèmes claniques des Umeda ${ }^{11}$. Il précise seulement que chaque clan fabrique un ou plusieurs masques et que chaque masque est " decorated with the distinguishing design or insignia of that clan. There may be 10 or more fish dancers at any time, though there are generally fewer than this." (Gell, 1975 : 188). Les Umeda étant constitués de six hameaux claniques répartis à égalité dans des moitiés homologues des moitiés

${ }^{10}$ Le totem du clan Menaneri (éteint depuis quelques générations), fondateur de la moitié féminine, est malheureusement oublié.

${ }^{11}$ Les Umeda sont désignés dans la région comme le lieu d'origine du rite Yangis; il y est nommé Ida (Gell 1975, Juillerat 1992). 
yafar, on peut émettre l'hypothèse que, si chaque clan peut fabriquer plus d'un masque, il ne devrait y avoir que six emblèmes différents. Concernant les Punda - un groupe waina (composé également de six hameaux claniques) s'intermariant avec les Umeda et, dans une moindre mesure, avec les Yafar qui leur ont emprunté le culte Yangis à la faveur de leurs liens matrimoniaux -, j'ai noté que les espèces naturelles des emblèmes de clans étaient différentes de celles des clans yafar, mais comme celles-ci sans corrélations entre elles.

En conclusion de son parcours historique et critique du totémisme, Claude Lévi-Strauss se déclare en accord avec Radcliffe-Brown sur le fait que :

" il est impossible de dissocier la forme du contenu. La forme n'est pas au dehors, mais au dedans. Pour percevoir la raison des dénominations animales, il faut les envisager concrètement ; car nous ne sommes pas libres de tracer une frontière, au delà de laquelle l'arbitraire régnerait. Le sens ne se décrète pas, il n'est nulle part s'il n'est partout. " (1962: 130)

L'exemple yafar met en évidence différents niveaux de sens, si l'on entend par sens d'une part la cohérence symbolique des totems entre eux pris au même niveau (ici moitiés ou clans), d'autre part la corrélation entre la relation entre totems (plantes, animaux, etc.) et la relation parallèle entre groupes sociaux (Ibid. : 18). De même qu'en Australie - comme l'avait signalé Radcliffe-Brown - certains totems de moitiés fondent leur cohérence dans leur appartenance à une même famille animale (par exemple, deux oiseaux) et leur différence dans l'écart signifiant entre ces deux espèces (faucon prédateur versus corneille charognarde chez certains Aborigènes, ou encore aigle de mer versus épervier pêcheur en NouvelleIrlande ${ }^{12}$ ), autant il parait évident que les deux palmiers totémiques des Yafar ont été " choisis " en fonction de leur appartenance à une même famille végétale et, simultanément, à leurs différences morphologiques complémentaires. Mais dans le cas yafar, la signification réelle n'est pas dans les corrélations entre totems, mais dans la dualité à laquelle ils 
renvoient, à savoir la complémentarité des fonctions paternelle et maternelle dans la procréation et la filiation. Ici, ce n'est pas la dualité en tant que telle qui fait sens par sa structure différentielle minimale, mais le signifié qui contient déjà la dualité.

En ce qui concerne le totémisme clanique, deux remarques s'imposent selon cette même ligne de réflexion. Premièrement, chaque groupe fut relié à une espèce à un moment de son histoire selon des critères qui sont aujourd'hui perdus pour la culture yafar (à l'exception éventuelle du totem " jeune" dont on a vu les connotations mythiques possibles). Deuxièmement, on ne note aucune corrélation sémantique entre les différents totems de clans, les espèces mentionnées étant indifféremment des plantes et des animaux (ces derniers de différents ordres). Cela ne signifie pas pour autant que le nombre de clans dans les sociétés de cette région soit indifférent; on a vu qu'un système à six groupes claniques et locaux est attesté chez les Waina ${ }^{13}$. Toujours est-il que les six " totems" claniques de base ne sont pas les mêmes dans les différentes sociétés et qu'il est vraisemblable que l'association à un esprit de la croissance (ogomô) lié à son tour à une espèce naturelle s'est produite, pour chaque clan, de façon contingente. Même si la symbolique du totem de clan eut un sens, aujourd'hui perdu, c'est le hasard des mouvements familiaux dans la région et de la " transplantation " des lignées d'une société à l'autre (Juillerat, 1986) qui est responsable de la disparité générale. 11 y a eu dispersion des totems claniques au gré de la dispersion démographique.

Cela nous amène à introduire la dimension historique pour parler du sens dans les organisations totémiques. Il arrive souvent que l'histoire bouleverse l'ordonnancement symbolique, mais il se peut aussi que certaines corrélations sémantiques ne se modifient plus en dépit du déplacement des personnes et des groupes. C'est ce qui semble s'être

${ }^{13}$ Chez les Sowanda, voisins occidentaux des Umeda, leur nombre est cependant plus élevé. 
produit avec les moitiés qui, dans toute cette région ${ }^{14}$, continuent de référer à l'opposition des sexes et plus particulièrement au couple parental. Dans toutes ces sociétés, les totems de moitiés sont en effet les mêmes ${ }^{15}$. Leur stabilité paraît défier les aléas de l'histoire locale. Il y a, selon nous, deux raisons à cela. La première est que le sens de la paire totémique des moitiés fait appel à des représentations psychiques universelles : la complémentarité des sexes, les invariants concernant la procréation ainsi que la dialectique produite par la double filiation à une mère et à un père. Une partie de ces représentations est inconsciente mais d'autant plus agissante dans les processus culturels de symbolisation ; on l'a vu avec la mise en scène rituelle des fils totémiques qui ne s'identifient au père social qu'à la condition d'une séparation préalable d'avec la mère parturiente. La seconde raison est que les moitiés ne sont pas des groupes de filiation (bien qu'ils soient composés de patriclans), mais des unités symboliques qui peuvent rester inchangées dans leurs références totémiques en dépit des modifications dont leurs clans pourraient être l'objet au sein de chaque moitié. Chez les Umeda et les Punda, lorsque la moitié d'un hameau clanique se trouve dépeuplée à la suite de l'extinction d'une lignée, on assigne à la moitié vacante quelques hommes jeunes de l'autre moitié du même hameau (appartenant en principe au même clan). Chez les Yafar - où les clans ne correspondent pas aux groupes locaux et où les semi-moitiés ne sont pas des moitiés de hameaux -, lorsqu'un rite Yangis était organisé, on rééquilibrait momentanément les moitiés en hommes dans le but d'un meilleur équilibre démographique entre elles et d'une meilleure complémentarité technique et symbolique du rite. Les unités duelles ne peuvent donc pas s'éteindre démographiquement. Elles pourront par contre disparaitre par l'abandon du culte masculin.

14 Cette région concerne les groupes de langue waina (Umeda, Punda, Sowanda, Waina-Wiyara), les Yafar (et autrefois les Potayneri et Wamuru) de langue amanab, peut-être aussi d'autres sociétés non étudiées à la périphérie de ces dernières et, notamment, en Irian Jaya (Indonésie) dont la frontière est toute proche et qui comprend une partie des deux aires linguistiques mentionnées.

${ }^{15}$ Gell n'a pas relevé les totems des moitiés umeda, mais ses matériaux ainsi que nos propres enquêtes ultérieures chez les Umeda, Punda et Sowanda confirment leur existence et leur conformité avec le totémisme yafar de moitiés. 
Cela dit, il nous reste encore un type de relation sémantique à établir : le lien que le rite Yangis construit dans son accomplissement sur la place du village entre le totémisme de moitié et le totémisme clanique. Dans la brève description du rite donnée plus haut, on a vu que la dualité totémique domine avec la danse copulatoire de la première nuit, puis à l'aube avec le Maître du Ciel et le Maître de la Terre (représentants de l'une et l'autre moitié) et avec les deux masques "Gelée de sagou ", enfin au terme de la représentation avec l'apparition des ifegê, des deux fils mythiques incarnant les jeunes pousses des palmiers totémiques et les hommes originels. Dans toutes ces séquences ce sont les moitiés qui sont à l'cuvre. Mais, dans la partie centrale du déroulement rituel, on a la danse (à sept protagonistes ${ }^{16}$ ) des masques claniques incarnant les esprits ogomô et les Poissons qui personnifient les fœtus en train de grandir dans le ventre maternel. L'entrée en scène des emblèmes claniques correspond à une participation des unités sociales réelles au processus de renouvellement des totems duels. Tout ce qui évoque la fécondation et la conception (séquence des gelées de sagou) renvoie à la dualité totémique, c'est-à-dire aux moitiés ; mais dès que l'on passe à la phase * croissance " (intra-utérine), c'est-à-dire à l'enfant en formation et à la nouvelle génération, alors chaque clan se doit d'y participer et a droit à en tirer profit. La santé démographique des groupes sociaux réels est dès lors concernée. $n$ y a plus : la société yafar étant patrilinéaire, la filiation idéologiquement privilégiée dans le rite totémique est la patrifiliation ${ }^{17}$.

Mais - surprise ! - voilà que les fruits de ce laborieux enfantement, les ifegê, sont à nouveau deux (et non pas un fils par clan), ont chacun un père initiateur et que leur masque, bien que du type "clanique ", ne porte aucun emblème (ou alors un emblème pluriclanique, indifférencié). Fruits de la participation plurielle des clans, les fils totémiques sont à nouveau inscrits dans la dualité. En fait rien d'étonnant à cela puisque la conclusion du rite met précisément en scène la situation générale de

${ }^{16}$ Autrefois sans doute huit, lorsque le clan fondateur " féminin " Menaneri existait encore.

${ }^{17}$ Les foetus totémiques sont directement issus, dans le rite, du mélange des deux spermes (sagous) totémiques lors de la danse des Gelées de sagou à l'aube. Chaque moitié, malgré l'opposition des genres, s'inscrit dans le même scénario reproductif. 
l'enfant mâle (pour les Yafar, seul un héros masculin peut incarner la société en général) face au problème de son autonomisation, c'est-à-dire de son renoncement à la mère, de son identification au père, enfin de sa propre transformation progrédiente en père potentiel. La problématique posée ici n'est plus celle de l'accroissement numérique ou de la santé du clan, mais bien celle du Sujet hors de tout groupe social particulier, du Sujet en devenir et de la Société en gestation. Dualité donc des ifegêe et des moitiés qu'ils représentent, mais en même temps ternarité dans la représentation du fils séparé de la mère génitrice par le père social, et aussi guidé par ce dernier vers la mère nourrice (tir des flèches vers le soleilsein). Yangis se clôt sur le registre ternaire de l'CEdipe. Le fait que le père mythique, autochâtré après la mort de sa compagne, devienne dans le rite un oncle maternel pose des questions sur lesquelles je n'ai pas la place de revenir ici (voir Juillerat, 1995). Disons seulement que le père géniteur, si important au début du rite, s'efface avec la mère parturiente pour laisser la place à un guide initiateur formant avec la mère nourrice un nouveau couple parental, socialisant et libérateur pour le Fils-Sujet en devenir. Au-delà de la reproduction des espèces totémiques, la signification fondamentale du totémisme yafar me parait résider dans cette transformation de la dualité parentale initiale en une ternarité socialisante qui permet d'échapper à l'originaire par la sortie du héros rituel hors du cycle totémique. Le personnage du père / oncle maternel (un pour chaque moitié et pour chaque ifegê) est assumé sur la place du village par un homme sans masque ni fard qui sort de la foule pour jouer son rôle, puis s'y fond à nouveau après le tir de sa flèche épointée. Ce père initiateur incarne, selon nous, l'affranchissement des origines totémiques de la Société. Dans la chronologie rituelle, il assure l'évasion hors du totémisme. I est le lien entre le présocial et le social. Quant aux ifegê, ils s'enfuient en courant dans les coulisses sitôt décochée leur propre flèche. Cet acte final (pour lequel j'ai proposé d'autres interprétations ailleurs ${ }^{19}$ ) pourrait signifier cette même rupture du lien totémique $:$ les " premiers hommes " fuient hors du scénario totémique, il s'enfuient vers leur nouveau statut d'êtres sociaux qui ne peut prendre forme que hors

18 Juillerat, 1992, 1995. 
du rite, dans la société elle-même. Le but du rite totémique est donc, en fin de compte, l'expression d'une émancipation hors des contraintes biologiques représentées par la reproduction des totems. C'est ce processus qui est sans cesse remis en marche à chaque célébration; chaque fois la société rejoue successivement sa dépendance et sa libération des origines. Le totémisme fournit le langage concret permettant de repenser et de revivre inlassablement cette transformation qui fonde l'identité individuelle et sociale de chaque homme yafar.

Bernard Juillerat C.N.R.S.

\section{Références bibliograpbiques}

Bateson, Gregory

1958 Naven. A Survey of the Problems suggested by a Composite Picture of the Culture of a New Guinea Tribe drawn from Three Points of View. Stanford, Stanford University Press (2d edition). Traduction française : La cérémonie du Naven. Paris, Editions de Minuit, 1971.

Gell, Alfred

1975 Metamorphosis of the Cassowaries. Umeda Society, Language and Ritual. London, The Athlone Press.

Harrison, Simon

1990 Stealing People's Names: History and Politics in a Sepik River Cosmology. Cambridge, Cambridge University Press.

Howells, William

1973 The Pacific Islanders. London, Weidenfeld \& Nicolson. 
Juillerat, Bernard

1986 Les enfants dusang. Société, reproduction et imaginaire en NouvelleGuinée. Paris, Maison des sciences de l'homme. - English translation : Children of the Blood. Society, Reproduction and Cosmology in New Guinea. Oxford - New York, Berg Publishers, 1996.

1992 'The Mother's Brother is the Breast' : Incest and Its Prohibition in the Yafar Yangis " et "Epilogue ", in B. Juillerat (ed.), Shooting the Sun. Ritual and Meaning in West Sepik. Washington, The Smithsonian Institution Press : 20-124 et 268-287.

1995 L'avènement du père. Rite, représentation, fantasme dans un culte mélanésien. Paris, CNRS - Maison des sciences de l'homme.

Lévi-Strauss, Claude

1962 Le totémisme aujourd'hui. Paris, Presses universitaires de France. 